\title{
Supporting Information for \\ Spent Caustic Regeneration in a Rotating Packed Bed: \\ Reaction and Separation Process Intensification
}

\author{
Yuan-Yuan Zhan, ${ }^{\dagger, \ddagger}$ Yuan-Fang Wan, ${ }^{\S}$ Meng-Jun Su, ${ }^{\dagger, \ddagger}$ \\ Yong Luo, ${ }^{\dagger, *}$ Guang-Wen Chu, ${ }^{\dagger, * *}$ Liang-Liang Zhang, ${ }^{\dagger, *}$ Jian-Feng Chen ${ }^{\dagger, \hbar}$
}

† State Key Laboratory of Organic-Inorganic Composites, Beijing University of

Chemical Technology, Beijing 100029, PR China

$\$$ Research Center of the Ministry of Education for High Gravity Engineering and Technology, Beijing University of Chemical Technology, Beijing 100029, PR China

$\S$ College of Mechanical and Electrical Engineering, Beijing University of Chemical Technology, Beijing 100029, PR China

* Tel: +8610 64446466; Fax: +861064434784.

*E-mail: luoyong@mail.buct.edu.cn; chugw@mail.buct.edu.cn 
The weights and bias values of the ANN have been listed as shown in Tables S1-S6.

Table S1. Weights of first hidden layer to input layer

\begin{tabular}{|c|c|c|c|c|c|c|}
\hline Weights & $\mathrm{I}_{1}$ & $\mathrm{I}_{2}$ & $\mathrm{I}_{3}$ & $\mathrm{I}_{4}$ & $\mathrm{I}_{5}$ & $\mathrm{I}_{6}$ \\
\hline $\mathrm{H}_{1}^{1}$ & -0.2519 & 0.0242 & 0.0330 & -0.1162 & 0.2079 & -0.2573 \\
\hline $\mathrm{H}_{2}^{1}$ & -0.2599 & 0.0312 & 0.0323 & -0.1057 & 0.2088 & -0.2688 \\
\hline $\mathrm{H}_{3}^{1}$ & -0.1478 & -0.0730 & 0.0462 & -0.3387 & 0.1629 & -0.0078 \\
\hline $\mathrm{H}_{4}^{1}$ & -0.2187 & -0.0143 & 0.0435 & -0.1779 & 0.2044 & -0.1926 \\
\hline $\mathrm{H}^{1}{ }_{5}$ & 0.0118 & -0.2888 & -0.3560 & 1.0746 & 0.6696 & -0.4445 \\
\hline $\mathrm{H}_{6}^{1}$ & -0.0600 & 0.1203 & -0.4458 & -0.0186 & 0.0962 & -0.2810 \\
\hline $\mathrm{H}_{7}^{1}$ & -0.2630 & 0.0338 & 0.0321 & -0.1018 & 0.2091 & -0.2731 \\
\hline $\mathrm{H}_{8}^{1}$ & -1.5397 & 0.7204 & 0.4711 & -0.4937 & 0.2252 & -0.8612 \\
\hline $\mathrm{H}_{9}^{1}$ & -0.2509 & 0.0233 & 0.0331 & -0.1176 & 0.2078 & -0.2557 \\
\hline $\mathrm{H}_{10}^{1}$ & -0.2484 & 0.0210 & 0.0335 & -0.1212 & 0.2075 & -0.2519 \\
\hline $\mathrm{H}_{11}^{1}$ & -0.2548 & 0.0268 & 0.0327 & -0.1123 & 0.2082 & -0.2616 \\
\hline $\mathrm{H}_{12}^{1}$ & 0.2185 & 0.1180 & -0.0654 & 0.6146 & -0.2291 & -0.1202 \\
\hline $\mathrm{H}_{13}^{1}$ & -0.2597 & 0.0310 & 0.0323 & -0.1060 & 0.2088 & -0.2685 \\
\hline $\mathrm{H}_{14}^{1}$ & 0.2563 & -0.0281 & -0.0325 & 0.1104 & -0.2084 & 0.2637 \\
\hline $\mathrm{H}_{15}^{1}$ & -0.2561 & 0.0280 & 0.0326 & -0.1106 & 0.2084 & -0.2635 \\
\hline $\mathrm{H}_{16}^{1}$ & 0.4411 & -0.7444 & -0.1701 & 0.2550 & -0.0591 & -0.2307 \\
\hline $\mathrm{H}^{1}{ }_{17}$ & -0.6221 & -0.8298 & -0.1738 & 0.6733 & 0.6080 & -1.2772 \\
\hline $\mathrm{H}_{18}^{1}$ & 0.2596 & -0.0309 & -0.0323 & 0.1061 & -0.2088 & 0.2684 \\
\hline $\mathrm{H}_{19}^{1}$ & -0.2982 & 0.7225 & -0.3131 & -0.1382 & 0.9480 & 0.1676 \\
\hline $\mathrm{H}_{20}^{1}$ & -0.2501 & 0.0226 & 0.0332 & -0.1187 & 0.2077 & -0.2546 \\
\hline
\end{tabular}


Table S2. Bias of first hidden layer to input layer

\begin{tabular}{|c|c|}
\hline & Bias \\
\hline $\mathrm{H}_{1}^{1}$ & -0.0960 \\
\hline $\mathrm{H}_{2}^{1}$ & -0.0970 \\
\hline $\mathrm{H}^{1}$ & -0.0474 \\
\hline $\mathrm{H}^{1}{ }_{4}$ & -0.0939 \\
\hline $\mathrm{H}_{5}^{1}$ & -0.1763 \\
\hline $\mathrm{H}^{1}$ & -0.0595 \\
\hline $\mathrm{H}^{1}$ & -0.0974 \\
\hline $\mathrm{H}^{1}$ & -0.1692 \\
\hline $\mathrm{H}_{8}^{1}$ & -0.0959 \\
\hline $\mathrm{H}_{10}^{1}$ & -0.0956 \\
\hline $\mathrm{H}_{11}^{1}$ & -0.0963 \\
\hline $\mathrm{H}_{12}^{1}$ & -0.0594 \\
\hline $\mathrm{H}_{13}^{1}$ & -0.0969 \\
\hline $\mathrm{H}_{14}^{1}$ & 0.0965 \\
\hline $\mathrm{H}_{15}^{1}$ & -0.0965 \\
\hline $\mathrm{H}_{16}^{1}$ & 0.3566 \\
\hline $\mathrm{H}_{17}^{1}$ & 0.2214 \\
\hline $\mathrm{H}_{18}^{1}$ & 0.0969 \\
\hline $\mathrm{H}_{19}^{1}$ & -0.2953 \\
\hline $\mathrm{H}_{20}^{1}$ & -0.0958 \\
\hline
\end{tabular}




\begin{tabular}{|c|c|c|c|c|c|c|c|c|c|c|c|c|c|c|c|c|c|c|c|c|}
\hline Jeights & $\mathrm{H}_{1}^{1}$ & $\mathrm{H}_{2}^{1}$ & $\mathrm{H}_{3}^{1}$ & $\mathrm{H}_{4}^{1}$ & $\mathrm{H}_{5}^{1}$ & $\mathrm{H}_{6}^{1}$ & $\mathrm{H}_{7}^{1}$ & $\mathrm{H}_{8}^{1}$ & $\mathrm{H}_{9}^{1}$ & $\mathrm{H}_{10}^{1}$ & $\mathrm{H}_{11}^{1}$ & $\mathrm{H}_{12}^{1}$ & $\mathrm{H}_{13}^{1}$ & $\mathrm{H}_{14}^{1}$ & $\mathrm{H}_{15}^{1}$ & $\mathrm{H}_{16}^{1}$ & $\mathrm{H}_{17}^{1}$ & $\mathrm{H}_{18}^{1}$ & $\mathrm{H}_{19}^{1}$ & $\mathrm{H}_{20}^{1}$ \\
\hline $\mathrm{H}_{1}^{2}$ & 0.0341 & 0.0340 & 0.0293 & 0.0337 & 0.0546 & 0.0676 & 0.0340 & 0.0259 & 0.0341 & 0.0341 & 0.0341 & -0.0113 & 0.0340 & -0.0344 & 0.0341 & 0.0276 & -0.0658 & -0.0340 & -0.0160 & 0.0341 \\
\hline $\mathrm{H}_{2}^{2}$ & -0.0341 & -0.0340 & -0.0293 & -0.0337 & -0.0546 & -0.0676 & -0.0340 & -0.0259 & -0.0341 & -0.0341 & -0.0341 & 0.0113 & -0.0340 & 0.0341 & -0.0341 & -0.0276 & 0.0658 & 0.0340 & 0.0160 & -0.0341 \\
\hline $\mathrm{H}_{3}^{2}$ & 0341 & -0.0340 & -0.0293 & -0.0337 & -0.0546 & -0.0676 & -0.0340 & -0.0259 & -0.0341 & 0.0341 & -0.0341 & 0.0113 & -0.0340 & 0.0341 & -0.0341 & -0.0276 & 0.0658 & 0.0340 & 0.0160 & -0.0341 \\
\hline $\mathrm{H}_{4}^{2}$ & -0.0341 & -0.0340 & -0.0293 & -0.0337 & 0.0546 & -0.0676 & -0.0340 & -0.0259 & -0.0341 & 0.0341 & -0.0341 & 0.0113 & -0.0340 & 0.0341 & -0.0341 & -0.0276 & 0.0658 & 0.0340 & 0.0160 & -0.0341 \\
\hline $\mathrm{H}_{5}^{2}$ & 0.0341 & 0.0340 & 0.0293 & 0.0337 & 0.0546 & 0.0676 & 0.0340 & 0.0259 & 0.0341 & 0.0341 & 0.0341 & -0.0113 & 0.0340 & -0.0341 & 0.0341 & 0.0276 & -0.0658 & -0.0340 & -0.0160 & 0.0341 \\
\hline $\mathrm{H}_{6}^{2}$ & -0.0341 & -0.0340 & -0.0293 & -0.0337 & -0.0546 & -0.0676 & -0.0340 & -0.0259 & -0.0341 & -0.0341 & -0.0341 & 0.0113 & -0.0340 & 0.0341 & -0.0341 & -0.0276 & 0.0658 & 0.0340 & 0.0160 & -0.0341 \\
\hline $\mathrm{H}_{7}^{2}{ }_{7}$ & 0.0341 & 0.0340 & 0.0293 & 0.0337 & 0.0546 & 0.0676 & 0.0340 & 0.0259 & 0.0341 & 0.0341 & 0.0341 & -0.0113 & 0.0340 & -0.0341 & 0.0341 & 0.0276 & -0.0658 & -0.0340 & -0.0160 & 0.0341 \\
\hline $\mathrm{H}_{8}^{2}$ & 0.0341 & 0.0340 & 0.0293 & 0.0337 & 0.0546 & 0.0676 & 0.0340 & 0.0259 & 0.0341 & 0.0341 & 0.0341 & -0.0113 & 0.0340 & -0.0341 & 0.0341 & 0.0276 & -0.0658 & -0.0340 & -0.0160 & 0.0341 \\
\hline $\mathrm{H}_{9}^{2}$ & & -0.0340 & -0.0293 & -0.0337 & -0.0546 & -0.0676 & -0.0340 & -0.0259 & -0.0341 & & -0.0341 & 0.0113 & -0.0340 & & & -0.0276 & 0.0658 & 0.0340 & 0.0160 & -0.0341 \\
\hline $\mathrm{H}_{10}^{2}$ & -0.0341 & -0.0340 & -0.0293 & -0.0337 & -0.0546 & -0.0676 & -0.0340 & -0.0259 & -0.0341 & -0.0341 & -0.0341 & 0.0113 & -0.0340 & 0.0341 & -0.0341 & -0.0276 & 0.0658 & 0.0340 & 0.0160 & -0.0341 \\
\hline $\mathrm{H}_{11}^{2}$ & 0.0341 & 0.0340 & 0.0293 & 0.0337 & 0.0546 & 0.0676 & 0.0340 & 0.0259 & 0.0341 & 0.0341 & 0.0341 & -0.0113 & 0.0340 & -0.0341 & 0.0341 & 0.0276 & -0.0658 & -0.0340 & -0.0160 & 0.0341 \\
\hline $\mathrm{H}_{12}^{2}$ & 0.2005 & 0.2057 & 0.1144 & 0.1764 & 0.4038 & 0.1656 & 0.2077 & 1.1162 & 0.1999 & 0.1982 & 0.2025 & -0.1388 & 0.2056 & -0.2034 & 0.2033 & -0.4208 & -0.4656 & -0.2056 & 0.3646 & 0.1994 \\
\hline $\mathrm{H}_{13}^{2}$ & -0.0341 & -0.0340 & -0.0293 & -0.0337 & -0.0546 & -0.0676 & -0.0340 & -0.0259 & -0.0341 & -0.0341 & -0.0341 & 0.0113 & -0.0340 & 0.0341 & -0.0341 & -0.0276 & 0.0658 & 0.0340 & 0.0160 & -0.0341 \\
\hline $\mathrm{H}_{14}^{2}$ & 0.0341 & 0.0340 & 0.0293 & 0.0337 & 0.0546 & 0.0676 & 0.0340 & 0.0259 & 0.0341 & 0.0341 & 0.0341 & -0.0113 & 0.0340 & -0.0341 & 0.0341 & 0.0276 & -0.0658 & -0.0340 & -0.0160 & 0.0341 \\
\hline $\mathrm{H}_{15}^{2}$ & 0341 & 0340 & 0.0293 & 0.0337 & 0.0546 & 0.0676 & 0.0340 & 0.0259 & 0.0341 & 0.0341 & 0.0341 & -0.0113 & 0.0340 & -0.0341 & 0.0341 & 0.0276 & -0.0658 & -0.0340 & -0.0160 & 0.0341 \\
\hline $\mathrm{H}_{16}^{2}$ & -0.0341 & -0.0340 & -0.0293 & -0.0337 & -0.0546 & -0.0676 & -0.0340 & -0.0259 & -0.0341 & -0.0341 & -0.0341 & 0.0113 & -0.0340 & 0.0341 & -0.0341 & -0.0276 & 0.0658 & 0.0340 & 0.0160 & -0.0341 \\
\hline $\mathrm{H}_{17}^{2}$ & -0.0341 & -0.0340 & -0.0293 & -0.0337 & -0.0546 & -0.0676 & -0.0340 & -0.0259 & -0.0341 & -0.0341 & -0.0341 & 0.0113 & -0.0340 & 0.0341 & -0.0341 & -0.0276 & 0.0658 & 0.0340 & 0.0160 & -0.0341 \\
\hline $\mathrm{H}_{18}^{2}$ & 0341 & 0340 & 0.0293 & 0.0337 & 0.0546 & 0.0676 & 0.0340 & 0.0259 & 0.0341 & 0.0341 & 0.0341 & -0.0113 & 0.0340 & -0.0341 & 0.0341 & 0.0276 & -0.0658 & -0.0340 & -0.0160 & 0.0341 \\
\hline $\mathrm{H}_{19}^{2}$ & -0.0341 & -0.0340 & -0.0293 & -0.0337 & -0.0546 & -0.0676 & -0.0340 & -0.0259 & -0.0341 & -0.0341 & -0.0341 & 0.0113 & -0.0340 & 0.0341 & -0.0341 & -0.0276 & 0.0658 & 0.0340 & 0.0160 & -0.0341 \\
\hline $\mathrm{H}_{20}^{2}$ & -0.2425 & -0.2359 & -0.3742 & -0.2889 & 0.7249 & 0.1810 & -0.2335 & 0.3333 & -0.2434 & -0.2457 & -0.2400 & 0.6205 & -0.2361 & 0.2387 & -0.2389 & 0.1376 & -0.7169 & 0.2361 & -0.9493 & -0.2441 \\
\hline $\mathrm{H}_{21}^{20}$ & 0341 & 0.0340 & 0.0293 & 0.0337 & 0.0546 & 0.0676 & 0.0340 & 0.0259 & 0.0341 & 0.0341 & 0.0341 & -0.0113 & 0.0340 & -0.0341 & 0.0341 & 0.0276 & -0.0658 & -0.0340 & -0.0160 & 0.0341 \\
\hline $\mathrm{H}_{22}^{2}$ & 0.0341 & 0.0340 & 0.0293 & 0.0337 & 0.0546 & 0.0676 & 0.0340 & 0.0259 & 0.0341 & 0.0341 & 0.0341 & -0.0113 & 0.0340 & -0.0341 & 0.0341 & 0.0276 & -0.0658 & -0.0340 & -0.0160 & 0.0341 \\
\hline $\mathrm{H}_{23}^{2}$ & -0.0341 & -0.0340 & -0.0293 & -0.0337 & -0.0546 & -0.0676 & -0.0340 & -0.0259 & -0.0341 & -0.0341 & -0.0341 & 0.0113 & -0.0340 & 0.0341 & -0.0341 & -0.0276 & 0.0658 & 0.0340 & 0.0160 & -0.0341 \\
\hline $\mathrm{H}_{24}^{2}$ & -0.0341 & -0.0340 & -0.0293 & -0.0337 & -0.0546 & -0.0676 & -0.0340 & -0.0259 & -0.0341 & -0.0341 & -0.0341 & 0.0113 & -0.0340 & 0.0341 & -0.0341 & -0.0276 & 0.0658 & 0.0340 & 0.0160 & -0.0341 \\
\hline $\mathrm{H}_{25}^{2}$ & -0.0341 & -0.0340 & -0.0293 & -0.0337 & -0.0546 & -0.0676 & -0.0340 & -0.0259 & -0.0341 & -0.0341 & -0.0341 & 0.0113 & -0.0340 & 0.0341 & -0.0341 & -0.0276 & 0.0658 & 0.0340 & 0.0160 & -0.0341 \\
\hline $\mathrm{H}_{26}^{2}$ & -0.0341 & -0.0340 & -0.0293 & -0.0337 & -0.0546 & -0.0676 & -0.0340 & -0.0259 & -0.0341 & -0.0341 & -0.0341 & 0.0113 & -0.0340 & 0.0341 & -0.0341 & -0.0276 & 0.0658 & 0.0340 & 0.0160 & -0.0341 \\
\hline $\mathrm{H}_{27}^{2}$ & -0.0341 & -0.0340 & -0.0293 & -0.0337 & -0.0546 & -0.0676 & -0.0340 & -0.0259 & -0.0341 & -0.0341 & -0.0341 & 0.0113 & -0.0340 & 0.0341 & -0.0341 & -0.0276 & 0.0658 & 0.0340 & 0.0160 & -0.0341 \\
\hline $\mathrm{H}_{28}^{2}$ & 0.0341 & 0.0340 & 0.0293 & 0.0337 & 0.0546 & 0.0676 & 0.0340 & 0.0259 & 0.0341 & 0.0341 & 0.0341 & -0.0113 & 0.0340 & -0.0341 & 0.0341 & 0.0276 & -0.0658 & -0.0340 & -0.0160 & 0.0341 \\
\hline $\mathrm{H}_{29}^{2}$ & 0.0050 & 0.0087 & 0.0310 & -0.0055 & -0.1362 & -0.0099 & 0.0102 & -1.0281 & 0.0046 & 0.0035 & 0.0064 & -0.2076 & 0.0086 & $\begin{array}{c}-0.0070 \\
\end{array}$ & 0.0070 & -0.6690 & 1.0076 & -0.0086 & 0.0024 & 0.0042 \\
\hline $\mathrm{H}_{30}^{2}$ & -0.0341 & -0.0340 & -0.0293 & -0.0337 & -0.0546 & -0.0676 & -0.0340 & -0.0259 & -0.0341 & -0.0341 & -0.0341 & 0.0113 & -0.0340 & 0.0341 & -0.0341 & -0.0276 & 0.0658 & 0.0340 & 0.0160 & -0.0341 \\
\hline $\mathrm{H}_{31}^{2}$ & & 0.0340 & 0.0293 & 0.0337 & 0.0546 & 0.0676 & 0.0340 & 0.0259 & 0.0341 & 0.0341 & 0.0341 & -0.0113 & 0.0340 & -0.0341 & 0.0341 & 0.0276 & -0.0658 & -0.0340 & -0.0160 & 0.0341 \\
\hline & 0. & 0.0340 & 0.0293 & 0.0337 & 0.0546 & 0.0676 & 0.0340 & 0.0259 & 0.0341 & 0.0341 & 0.0341 & -0.0113 & 0.0340 & -0.0341 & 0.0341 & 0.0276 & -0.0658 & -0.0340 & -0.0160 & 0.0341 \\
\hline $\mathrm{H}_{33}^{2}$ & -0.2692 & -0.2872 & 0.0813 & -0.1735 & -0.4413 & -0.2500 & -0.2940 & -1.0577 & -0.2668 & -0.2609 & -0.2758 & -0.2776 & -0.2866 & 0.2792 & -0.2788 & 0.0628 & -0.6045 & 0.2865 & 0.3293 & -0.2650 \\
\hline $\mathrm{H}_{34}^{2}$ & 3341 & 0.0340 & 0.0293 & 0.0337 & 0546 & 0.0676 & 0.0340 & 0.0259 & & 0.0341 & 0.0341 & & 0.0340 & -0.0341 & 0.0341 & 0.0276 & -0.0658 & -0.0340 & -0.0160 & 0.0341 \\
\hline $\mathrm{H}_{35}^{2}$ & 0.0341 & 0.0340 & 0.0293 & 0.0337 & 0.0546 & 0.0676 & 0.0340 & 0.0259 & 0.0341 & 0.0341 & 0.0341 & -0.0113 & 0.0340 & -0.0341 & 0.0341 & 0.0276 & -0.0658 & -0.0340 & -0.0160 & 0.0341 \\
\hline $\mathrm{H}_{36}^{2}$ & -0.0341 & -0.0340 & -0.0293 & -0.0337 & -0.0546 & -0.0676 & -0.0340 & -0.0259 & -0.0341 & -0.0341 & -0.0341 & 0.0113 & -0.0340 & 0.0341 & -0.0341 & -0.0276 & 0.0658 & 0.0340 & 0.0160 & -0.0341 \\
\hline $\mathrm{H}_{37}^{2}$ & 341 & 0.0340 & 0.0293 & & 0546 & 0.0676 & 0.0340 & 0.0259 & & $\begin{array}{l}0.0341 \\
\end{array}$ & & & & -0.03 & & 0.0276 & -0.0658 & -0.0340 & -0.0160 & 0.0341 \\
\hline $\mathrm{H}_{38}^{2}$ & 0.0341 & 0.0340 & 0.0293 & 0.0337 & 0.0546 & 0.0676 & 0.0340 & 0.0259 & 0.0341 & 0.0341 & 0.0341 & -0.0113 & 0.0340 & -0.0341 & 0.0341 & 0.0276 & -0.0658 & -0.0340 & -0.0160 & 0.0341 \\
\hline $\mathrm{H}_{39}^{2}$ & 0.0341 & 0.0340 & 0.0293 & 0.0337 & 0.0546 & 0.0676 & 0.0340 & 0.0259 & 0.0341 & 0.0341 & 0.0341 & -0.0113 & 0.0340 & -0.0341 & 0.0341 & 0.0276 & -0.0658 & -0.0340 & -0.0160 & 0.0341 \\
\hline $\mathrm{H}_{40}^{2}$ & -0.0341 & -0.0340 & -0.0293 & & -0.0546 & -0.0676 & -0.0340 & -0.0259 & & -0.0341 & -0.0341 & & -0.0340 & & & -0.0276 & 0.0658 & 0.0340 & 0.0160 & -0.0341 \\
\hline $\mathrm{H}_{41}^{2}$ & -0.0341 & -0.0340 & -0.0293 & -0.0337 & .0546 & -0.0676 & -0.0340 & -0.0259 & -0.03 & -0.03 & -0.0341 & 0.01 & -0.0340 & 0.03 & -0.0341 & -0.0276 & 0.0658 & 0.0340 & 0.0160 & -0.0341 \\
\hline $\mathrm{H}_{42}^{2}$ & -0.0341 & -0.0340 & -0.0293 & -0.0337 & -0.0546 & -0.0676 & -0.0340 & -0.0259 & -0.0341 & -0.0341 & -0.0341 & 0.0113 & -0.0340 & 0.0341 & -0.0341 & -0.0276 & 0.0658 & 0.0340 & 0.0160 & -0.0341 \\
\hline $\mathrm{H}_{43}^{2}$ & -0.0341 & -0.0340 & -0.0293 & & -0.0546 & -0.0676 & -0.0340 & & & & & & & & & -0.0276 & 0.0658 & 0.0340 & 0.0160 & -0.0341 \\
\hline $\mathrm{H}_{44}^{2}$ & $\begin{array}{c}-0.0341 \\
\end{array}$ & -0.0340 & -0.0293 & .0337 & -0.0546 & -0.0676 & -0.0340 & -0.0259 & -0.0341 & -0.03 & -0.0341 & & -0.0340 & 0.03 & -0.0341 & -0.0276 & 0.0658 & 0.03 & 0.0160 & \begin{tabular}{|c|}
-0.0341 \\
\end{tabular} \\
\hline $\mathrm{H}_{45}^{2}$ & -0.0341 & -0.0340 & -0.0293 & -0.0337 & -0.0546 & -0.0676 & -0.0340 & -0.0259 & -0.0341 & -0.0341 & -0.0341 & 0.0113 & -0.0340 & 0.0341 & -0.0341 & -0.0276 & 0.0658 & 0.0340 & 0.0160 & -0.0341 \\
\hline $\mathrm{H}_{46}^{2}$ & 0.0341 & 0.0340 & 0.0293 & & 0.0546 & 0.0676 & 0.0340 & 0.0259 & & 0.0341 & 0.0341 & -0.0113 & & -0.0341 & 0.0341 & 0.0276 & -0.0658 & -0.0340 & -0.0160 & 0.0341 \\
\hline $\mathrm{H}_{47}^{2}$ & 0.0341 & 0.0340 & 0.0293 & 0.0337 & 0.0546 & 0.0676 & 0.0340 & 0.0259 & 0.0341 & 0.0341 & 0.0341 & -0.0113 & 0.0340 & -0.0341 & 0.0341 & 0.0276 & -0.0658 & -0.0340 & -0.0160 & 0.0341 \\
\hline $\mathrm{H}_{48}^{2}$ & 0.0341 & 0.0340 & 0.0293 & 0.0337 & 0.0546 & 0.0676 & 0.0340 & 0.0259 & 0.0341 & 0.0341 & 0.0341 & -0.0113 & 0.0340 & -0.0341 & 0.0341 & 0.0276 & -0.0658 & -0.0340 & -0.0160 & 0.0341 \\
\hline $\mathrm{H}_{49}^{20}$ & $\begin{array}{c}-0.0341 \\
\end{array}$ & -0.0340 & -0.0293 & -0.0337 & -0.0546 & -0.0676 & -0.0340 & -0.0259 & -0.0341 & -0.0341 & -0.0341 & 0.0113 & -0.0340 & 0.0341 & -0.0341 & -0.0276 & 0.0658 & 0.0340 & 0.0160 & -0.0341 \\
\hline $\mathrm{H}_{50}^{2}$ & -0.0341 & -0.0340 & -0.0293 & -0.0337 & -0.0546 & -0.0676 & -0.0340 & -0.0259 & -0.0341 & $\begin{array}{l}-0.0341 \\
\end{array}$ & -0.0341 & 0.0113 & $\begin{array}{l}-0.0340 \\
\end{array}$ & 0.0341 & -0.0341 & -0.0276 & 0.0658 & 0.0340 & 0.0160 & -0.0341 \\
\hline
\end{tabular}


Table S4. Bias of second hidden layer to first hidden layer

\begin{tabular}{|c|c|}
\hline & Bias \\
\hline $\mathrm{H}_{1}^{2}$ & 0.0155 \\
\hline $\mathrm{H}_{2}^{2}$ & -0.0155 \\
\hline $\mathrm{H}_{3}^{2}$ & -0.0155 \\
\hline $\mathrm{H}_{4}^{2}$ & -0.0155 \\
\hline $\mathrm{H}^{2}{ }_{5}$ & 0.0155 \\
\hline $\mathrm{H}_{6}^{2}$ & -0.0155 \\
\hline $\mathrm{H}_{7}^{2}$ & 0.0155 \\
\hline $\mathrm{H}_{8}^{2}$ & 0.0155 \\
\hline $\mathrm{H}_{9}^{2}$ & -0.0155 \\
\hline $\mathrm{H}_{10}^{2}$ & -0.0155 \\
\hline $\mathrm{H}^{2}{ }_{11}$ & 0.0155 \\
\hline $\mathrm{H}_{12}^{2}$ & -0.0886 \\
\hline $\mathrm{H}_{13}^{2}$ & -0.0155 \\
\hline $\mathrm{H}_{14}^{2}$ & 0.0155 \\
\hline $\mathrm{H}_{15}^{2}$ & 0.0155 \\
\hline $\mathrm{H}_{16}^{2}$ & -0.0155 \\
\hline $\mathrm{H}_{17}^{2}$ & -0.0155 \\
\hline $\mathrm{H}_{18}^{2}$ & 0.0155 \\
\hline $\mathrm{H}_{19}^{2}$ & -0.0155 \\
\hline $\mathrm{H}_{20}^{2}$ & -0.0209 \\
\hline $\mathrm{H}_{21}^{2}$ & 0.0155 \\
\hline $\mathrm{H}_{22}^{2}$ & 0.0155 \\
\hline $\mathrm{H}_{23}^{2}$ & -0.0155 \\
\hline $\mathrm{H}_{24}^{2}$ & -0.0155 \\
\hline $\mathrm{H}_{25}^{2}$ & -0.0155 \\
\hline $\mathrm{H}_{26}^{2}$ & -0.0155 \\
\hline $\mathrm{H}_{27}^{2}$ & -0.0155 \\
\hline $\mathrm{H}_{28}^{2}$ & 0.0155 \\
\hline $\mathrm{H}_{29}^{2}$ & 0.0822 \\
\hline $\mathrm{H}_{30}^{2}$ & -0.0155 \\
\hline $\mathrm{H}_{31}^{2}$ & 0.0155 \\
\hline $\mathrm{H}_{32}^{2}$ & 0.0155 \\
\hline $\mathrm{H}_{33}^{2}$ & 0.1653 \\
\hline $\mathrm{H}_{34}^{2}$ & 0.0155 \\
\hline $\mathrm{H}_{35}^{2}$ & 0.0155 \\
\hline $\mathrm{H}_{36}^{2}$ & -0.0155 \\
\hline $\mathrm{H}_{37}^{2}$ & 0.0155 \\
\hline $\mathrm{H}_{38}^{2}$ & 0.0155 \\
\hline $\mathrm{H}_{39}^{2}$ & 0.0155 \\
\hline $\mathrm{H}_{40}^{2}$ & -0.0155 \\
\hline $\mathrm{H}_{41}^{2}$ & -0.0155 \\
\hline $\mathrm{H}_{42}^{2}$ & -0.0155 \\
\hline $\mathrm{H}_{43}^{2}$ & -0.0155 \\
\hline $\mathrm{H}_{44}^{2}$ & -0.0155 \\
\hline $\mathrm{H}_{45}^{2}$ & -0.0155 \\
\hline $\mathrm{H}_{46}^{2}$ & 0.0155 \\
\hline $\mathrm{H}_{47}^{2}$ & 0.0155 \\
\hline $\mathrm{H}_{48}^{2}$ & 0.0155 \\
\hline $\mathrm{H}_{49}^{2}$ & -0.0155 \\
\hline $\mathrm{H}_{50}^{2}$ & -0.0155 \\
\hline
\end{tabular}


Table S5. Weights of output layer to second hidden layer

\begin{tabular}{|c|c|c|}
\hline Weights & $\overline{\mathrm{O}_{1}}$ & $\mathrm{O}_{2}$ \\
\hline $\mathrm{H}_{1}^{2}$ & 0.1366 & 0.1084 \\
\hline $\mathrm{H}_{2}^{2}$ & -0.1366 & -0.1084 \\
\hline $\mathrm{H}_{3}^{2}$ & -0.1366 & -0.1084 \\
\hline $\mathrm{H}_{4}^{2}$ & -0.1366 & -0.1084 \\
\hline $\mathrm{H}^{2}{ }_{5}$ & 0.1366 & 0.1084 \\
\hline $\mathrm{H}_{6}^{2}$ & -0.1366 & -0.1084 \\
\hline $\mathrm{H}^{2}{ }_{7}$ & 0.1366 & 0.1084 \\
\hline $\mathrm{H}_{8}^{2}$ & 0.1366 & 0.1084 \\
\hline $\mathrm{H}_{9}^{2}$ & -0.1366 & -0.1084 \\
\hline $\mathrm{H}_{10}^{2}$ & -0.1366 & -0.1084 \\
\hline $\mathrm{H}_{11}^{2}$ & 0.1366 & 0.1084 \\
\hline $\mathrm{H}_{12}^{2}$ & -0.5458 & 0.7574 \\
\hline $\mathrm{H}_{13}^{2}$ & -0.1366 & -0.1084 \\
\hline $\mathrm{H}_{14}^{2}$ & 0.1366 & 0.1084 \\
\hline $\mathrm{H}_{15}^{2}$ & 0.1366 & 0.1084 \\
\hline $\mathrm{H}_{16}^{2}$ & -0.1366 & -0.1084 \\
\hline $\mathrm{H}_{17}^{2}$ & -0.1366 & -0.1084 \\
\hline $\mathrm{H}_{18}^{2}$ & 0.1366 & 0.1084 \\
\hline $\mathrm{H}_{19}^{2}$ & -0.1366 & -0.1084 \\
\hline $\mathrm{H}_{20}^{2}$ & 0.4537 & 1.5489 \\
\hline $\mathrm{H}_{21}^{2}$ & 0.1366 & 0.1084 \\
\hline $\mathrm{H}_{22}^{2}$ & 0.1366 & 0.1084 \\
\hline $\mathrm{H}_{23}^{2}$ & -0.1366 & -0.1084 \\
\hline $\mathrm{H}_{24}^{2}$ & -0.1366 & -0.1084 \\
\hline $\mathrm{H}_{25}^{2}$ & -0.1366 & -0.1084 \\
\hline $\mathrm{H}_{26}^{2}$ & -0.1366 & -0.1084 \\
\hline $\mathrm{H}_{27}^{2}$ & -0.1366 & -0.1084 \\
\hline $\mathrm{H}_{28}^{2}$ & 0.1366 & 0.1084 \\
\hline $\mathrm{H}_{29}^{2}$ & -1.1403 & 0.6863 \\
\hline $\mathrm{H}_{30}^{2}$ & -0.1366 & -0.1084 \\
\hline $\mathrm{H}_{31}^{2}$ & 0.1366 & 0.1084 \\
\hline $\mathrm{H}_{32}^{2}$ & 0.1366 & 0.1084 \\
\hline $\mathrm{H}_{33}^{2}$ & -0.8348 & -0.6853 \\
\hline $\mathrm{H}_{34}^{2}$ & 0.1366 & 0.1084 \\
\hline $\mathrm{H}_{35}^{2}$ & 0.1366 & 0.1084 \\
\hline $\mathrm{H}_{36}^{2}$ & -0.1366 & -0.1084 \\
\hline $\mathrm{H}_{37}^{2}$ & 0.1366 & 0.1084 \\
\hline $\mathrm{H}_{38}^{2}$ & 0.1366 & 0.1084 \\
\hline $\mathrm{H}_{39}^{2}$ & 0.1366 & 0.1084 \\
\hline $\mathrm{H}_{40}^{2}$ & -0.1366 & -0.1084 \\
\hline $\mathrm{H}_{41}^{2}$ & -0.1366 & -0.1084 \\
\hline $\mathrm{H}_{42}^{2}$ & -0.1366 & -0.1084 \\
\hline $\mathrm{H}_{43}^{2}$ & -0.1366 & -0.1084 \\
\hline $\mathrm{H}_{44}^{2}$ & -0.1366 & -0.1084 \\
\hline $\mathrm{H}_{45}^{2}$ & -0.1366 & -0.1084 \\
\hline $\mathrm{H}_{46}^{2}$ & 0.1366 & 0.1084 \\
\hline $\mathrm{H}_{47}^{2}$ & 0.1366 & 0.1084 \\
\hline $\mathrm{H}_{48}^{2}$ & 0.1366 & 0.1084 \\
\hline $\mathrm{H}_{49}^{2}$ & -0.1366 & -0.1084 \\
\hline $\mathrm{H}_{50}^{2}$ & -0.1366 & -0.1084 \\
\hline
\end{tabular}


Table S6. Bias of output layer to second hidden layer

\begin{tabular}{|c|c|}
\hline & Bias \\
\hline $\mathrm{O}_{1}$ & 0.0320 \\
\hline $\mathrm{O}_{2}$ & 0.1415 \\
\hline
\end{tabular}

